\title{
Labyrinthe
}

\section{Empires : la logique de la domination mondiale - de la Rome antique aux États-Unis}

\author{
Herfried Münkler \\ Traducteur : Thierry Carpent et Tobias Mörike
}

\section{OpenEdition \\ Journals}

\section{Édition électronique}

URL : http://journals.openedition.org/labyrinthe/4079

DOI : $10.4000 /$ labyrinthe.4079

ISSN : 1950-6031

Éditeur

Hermann

Édition imprimée

Date de publication : 28 août 2010

Pagination : 39-56

ISBN : 9782705669973

Référence électronique

Herfried Münkler, «Empires : la logique de la domination mondiale - de la Rome antique aux États-

Unis », Labyrinthe [En ligne], 35 | 2010 (2), mis en ligne le 27 juillet 2012, consulté le 30 avril 2019. URL : http://journals.openedition.org/labyrinthe/4079; DOI : 10.4000/labyrinthe.4079 


\title{
Empires : \\ la logique de la domination mondiale - de la Rome antique aux États-Unis
}

\author{
Herfried MÜNKLER \\ Contact : herfried.muenkler@sowi.hu-berlin.de
}

(c) 2005 Rowohlt Verlag. La version originale de ce texte est parue dans Imperien : die Logik der Weltherrschaft - vom Alten Rom bis zu den Vereinigten Staaten, Berlin, Rowohlt, 2005, p. 7-21. Reproduction sur autorisation. Version française revue et corrigée par l'auteur.

\section{Préface}

Depuis la deuxième moitié du $\mathrm{xx}^{\mathrm{e}}$ siècle, plus personne, dans la recherche allemande, ne s'est particulièrement intéressé à la théorie et à l'histoire des empires. C'est seulement lors de l'effondrement de l'Union soviétique que l'intérêt pour ce sujet s'est brièvement ravivé, assorti du soulagement à constater que l'histoire des empires, qui remontait à l'époque des premières grandes civilisations, était définitivement terminée. Cette perception a radicalement changé au cours des dernières années lorsqu'est apparu au grand jour le rôle nouveau que les États-Unis assumaient sur la scène internationale. On s'est mis d'un seul coup à parler d'Empire américain et, depuis, la critique exercée à l'encontre de l'action des États-Unis dans la politique mondiale revêt des traits fortement hostiles à l'idée d'empire. Il est vrai que par le passé, on a souvent accusé les États-Unis d'impérialisme, comme par exemple pendant la Guerre du Vietnam ou à l'occasion de leurs interventions militaires en Amérique latine ou dans le golfe Persique. Cependant, ces accusations étaient toujours dirigées contre des décisions ou des actions ponctuelles du gouvernement américain, tandis que l'attitude fondamentalement hostile à l'idée d'empire a pour cible la prépondérance des États-Unis et leurs prétentions à la suprématie en tant que telles. C'est bien différent. 
« Avant le 11 septembre, l'attitude des mouvements nord-américains et européens face à la mondialisation était la suivante : ils avaient plus ou moins la même idée de l'empire que (Toni Negri et moi). Pourquoi dis-je cela ? Parce que s'ils avaient pensé que la Maison Blanche, l'État fédéral ou le gouvernement des ÉtatsUnis étaient directement responsables de tous les problèmes de domination dans le monde, ils auraient dû manifester devant la Maison Blanche chaque semaine. Mais au lieu de cela, ils ont reconnu qu'il y avait une nouvelle structure de pouvoir, et de nouveaux ennemis. Et ils expérimentaient, en identifiant de possibles ennemis : "Allez, essayons le G8", "Essayons la Banque Mondiale et le FMI", "Essayons l'OMC". D'une certaine manière, toutes ces expériences étaient inadéquates, mais elles configuraient un réseau de pouvoirs qui de fait constituait la nouvelle structure du pouvoir. Nous ne disons rien d'autre, je pense. » Michael Hardt, " Empire », Conversations with History, 12 mars 2004, URL : http://globetrotter.berkeley.edu/people4/Hardt/hardtcon7.html (visité le 19 mai 2010)
L'existence d'une puissance prépondérante impériale estelle nécessaire à la sécurité de la communauté mondiale ? Ou bien le fait qu' une telle puissance impériale soit prépondérante perturbe-t-il gravement l'ordre mondial, au point qu'il vaudrait mieux dès lors qu'elle n'existe pas? C'est en principe autour de cette question que tourne le débat mené avant la dernière guerre du Golfe. En

effet, au cours des dernières années, la communauté internationale réunie au sein de l'ONU n'a cessé d'avoir recours aux capacités de cette puissance impériale prépondérante. Mais on n'a pas voulu admettre que, loin d'intervenir de façon désintéressée, les ÉtatsUnis voulaient obtenir des privilèges en échange de ces sollicitations. Les agacements qui en ont résulté étaient eux aussi une conséquence du fait que, depuis longtemps, on n'avait plus réfléchi à ce qu'étaient les fonctions et les exigences d'un empire.

Les empires sont plus que de grands États, ils se meuvent dans un monde qui leur est propre. Les États sont partie intégrante d'un

« L'enjeu est de prêter attention à la manière dont des régimes d'exception [...] sont définis et défendus de nos jours. Peut-être l'invocation de l'empire est-elle un moyen de pénétrer par effraction dans le jeu de normes internationales consacré ces dernières décennies, qu'il s'agisse d'universalité de la souveraineté ou de respect envers le code international de bonne conduite. Parler d'empire, c'est modifier la donne, sur la base d'une distinction hiérarchique entre les États ou les peuples qui peuvent prétendre à la souveraineté et à l'auto-détermination, et ceux qui ne les méritent pas. »

Craig Calhoun, Frederick Cooper, Kevin W. Moore (dir.), Lessons of Empire. Imperial Histories and American Power, New York, The New Press, 2006, « Introduction », p. 3. 
ordre qu'ils ont créé ensemble avec d'autres États et dont ils ne peuvent donc disposer seuls. Les empires en revanche se comprennent comme les créateurs et les garants d'un ordre qui, en dernier ressort, dépend d'eux et qu'ils doivent défendre contre la menace constante que représente pour eux l'irruption du chaos. Un regard jeté sur l'histoire, et pas seulement sur celle des États-Unis, montre que des expressions comme «l'axe du Mal » ou «les avant-postes de la tyrannie » n'ont rien de nouveau ni de particulier. Au contraire, ces expressions parcourent l'histoire des empires comme un fil rouge.

À la peur que le chaos ne fasse irruption et à la fonction autoproclamée de défenseur de l'ordre contre le désordre, du bien contre le mal, de laquelle l'empire se voit revêtu et par laquelle il se donne une légitimité, correspond la mission impériale qui constitue également une justification fondamentale de la constitution d'un empire mondial : la civilisation doit être propagée ; ce sont l'adoption de l'ordre social socialiste, la protection des droits de l'Homme ou la promotion de la démocratie qui sont en jeu. Alors que les États s'arrêtent aux frontières des autres États et laissent ceux-ci régler eux-mêmes leurs affaires intérieures, les empires s'ingèrent dans les affaires des autres afin d'accomplir leur mission. C'est pourquoi les empires peuvent être à l'origine de processus de changement particulièrement puissants, tandis que l'ordre des États est plutôt marqué par un conservatisme structurel.

Si l'on considère les choses de ce point de vue, ce qui est devenu une évidence sous l'influence des théories de l'impérialisme, à savoir que l'idéal à atteindre consisterait en un ordre global d'États égaux entre eux sans acteur impérial, est rien moins que sûr. Dans l'ordonnancement politique de l'espace européen tel qu'il s'est développé après la chute de l'empire romain, il n'existait plus de force impériale capable d'agir durablement ; en revanche, il n'a pas manqué de prétendants à ce rôle, lesquels ont échoué très rapidement. Il n'en a pas été ainsi ailleurs - même si l'on fait abstraction du fait qu'indubitablement, les Européens ont établi des empires sur d'autres continents. C'est avant tout en Asie que s'est imposé un ordre politique dans lequel les empires se sont entourés d'une couronne d'États clients ; en conséquence, ces espaces étaient organisés autour d'un centre fort, tandis qu'un système polycentrique varié s'est développé en Europe.

L'image que nous nous faisons des empires est marquée par l'idée selon laquelle la périphérie aurait été exploitée au maximum par le centre, qu'elle 
se serait appauvrie et le centre enrichi. De tels empires ont effectivement toujours existé, mais ils n'ont jamais duré très longtemps. Au bout d'un certain temps, la résistance contre le centre a pris le dessus et les dépenses liées au maintien de la domination ont surpassé les gains que celui-ci tirait de la périphérie. En revanche, les empires ayant perduré sont ceux qui ont investi dans la périphérie et veillé ainsi à ce que, tout autant que le centre, la périphérie trouve son intérêt dans le maintien de l'empire.

Ce dont il sera question dans ce livre, ce sont les types de la domination impériale, les formes de l'expansion et de la consolidation, et les modalités selon lesquelles s'est réalisée la création d'empires. Notre investigation ne se limite cependant pas à distinguer les empires maritimes des empires continentaux, les empires commerciaux des empires militaires, les ordres impériaux qui se développent par le biais d'un contrôle des espaces de ceux qui consistent essentiellement à contrôler les flux (d'hommes, de biens ou de capitaux); au-delà de tout cela, elle s'intéresse également à la rationalité des acteurs et, partant, à la logique présidant à la domination du monde. Il s'agit également d'établir des pronostics sur la durée et la stabilité de l'empire américain et de réfléchir à la façon dont doit se constituer une Europe susceptible d'un côté de s'affirmer comme force politique autonome à l'égal des États-Unis, et de l'autre de consolider ses marges instables et chancelantes tout en exerçant une action positive sur ses voisins. Une telle Europe ne pourra pas faire autrement que de reprendre à son compte des caractéristiques impériales et de développer des capacités impériales — si l'on y prête attention, ce processus est déjà en route. Cela suppose assurément que l'on ne perçoive pas les activités impériales comme a priori mauvaises ou répréhensibles, mais qu'on les considère comme une autre façon de traiter les problèmes que celles qui reviennent aux États et à d'autres formes d'organisation du politique.

Il ne faut pas confondre cela avec la réhabilitation des anciens empires coloniaux. Le mythe fondateur des États-Unis, c'est d'avoir mené un combat permettant de se libérer d'un tel empire colonial ; les Européens se perçoivent comme ayant exercé une telle forme de domination d'espaces extra-européens avant de s'en défaire ultérieurement. On est cependant en droit de douter du modèle misant sur l'égalité et la réciprocité des États, et de sa capacité à relever les défis qui s'annoncent au cours des prochaines décennies. Car c'est la faillite ou le déclin des États qui provoque l'intervention et l'apparition d'empires. 
Nombreux sont en revanche ceux qui vont objecter que l'opposition entre État et empire ne permet pas de faire le tour du sujet, et qui vont en conséquence énumérer leurs représentations de ce qu'est un ordre politique idéal - mais qui, ce faisant, s'éloignent toujours plus de la réalité. Le regard que nous portons sur l'histoire montre que les notions d'État et d'empire ont toujours permis de traiter les modèles d'ordre politique de façon exhaustive, à condition toutefois d'utiliser ces termes le plus largement possible et de ne pas inventer une notion spécifique pour chaque cas particulier d'État ou d'empire. Ce que nous nous proposons ici, c'est de sonder ce que la notion d'empire peut apporter. En présentant la façon dont les empires se sont constitués et ont décliné, nous entrons, du point de vue de la recherche, sur un terrain resté longtemps en jachère.

Berlin, février 2005

$$
* * *
$$

\section{Chapitre 1 Qu'est-ce qu'un empire?}

En Europe, les débats menés au sujet de la dernière guerre d'Irak, les arrière-pensées possibles et les buts cachés de cette nouvelle intervention militaire des États-Unis dans la région pétrolifère du Golfe, et plus généralement le rôle des États-Unis dans la région du Golfe et en Asie centrale (sans oublier les profondes dissensions caractérisant les relations transatlantiques) ont aiguisé le regard porté sur la constitution d'un nouvel ordre mondial après la fin de la confrontation Est-Ouest. Une redéfinition de la position américaine dans l'ordre politique du monde est apparue du fait du refus notoire des États-Unis de s'associer aux traités internationaux, qu'il s'agisse du protocole de Kyoto ou du Tribunal pénal international de La Haye. Après avoir été constamment problématiques dans les décennies passés, les relations entre les ÉtatsUnis et l'ONU ont été fondamentalement remises en question depuis que, dans sa mémorable intervention du 12 novembre 2002 devant l'Assemblée générale des Nations-Unies, le président George W. Bush a brandi la menace selon laquelle les États-Unis allaient résoudre seuls 
certains problèmes de sécurité urgents si l'organisation mondiale se révélait incapable de le faire.

La Troisième Guerre du Golfe, engagée au printemps 2003, a montré qu'il ne s'agissait pas là de vains mots. Deux interprétations des nouveaux rapports établis entre le Conseil de sécurité de l'ONU et les États-Unis étaient possibles : les États-Unis cherchaient soit à instrumentaliser celui-ci en le chargeant de légitimer leur intervention, soit à s'émanciper de leur rôle de bras armé de l'organisation mondiale : cessant de mettre leur appareil militaire coûteux et sophistiqué au service de la communauté internationale, ils allaient dès lors l'utiliser au profit de leurs buts et de leurs intérêts propres. Les conflits intervenus dans la période précédant la guerre en Irak ont également donné lieu à une controverse sur la question de savoir qui, des États-Unis ou des Nations Unies, allait instrumentaliser l'autre'.

L'architecture de sécurité européenne sur laquelle on s'était jusqu'alors reposé en Allemagne semblait s'être tout autant fragilisée. De façon imperceptible, l'OTAN a cessé dans le courant des années 1990 d'être une alliance fondée sur une base consultative pour se transformer en instrument de contrôle exercé par les États-Unis sur l'Europe. Et là où cette structure s'est montrée trop encombrante pour la politique américaine, elle a été remplacée sans autre forme de procès par une « coalition of the willing ». Par rapport à l'époque de la Guerre froide, la dépendance effective de l'Europe à l'égard des États-Unis s'est plutôt accrue qu'elle n'a diminué. Ceux qui refusent de participer à la réalisation des plans américains doivent s'attendre soit à faire l'objet de pressions politiques et économiques, soit à être couverts de sarcasmes. Il n'y a aucun problème à ce que ceux qui en revanche acceptent de s'engager aux côtés des Américains le fassent - mais alors, aux conditions posées par ces derniers et sans avoir la moindre influence sur des décisions politiques fondamentales : même la Grande-Bretagne, le principal allié des États-Unis, en a fait plus d'une fois l'expérience. Les problèmes dans lesquels les États-Unis se sont trouvés embourbés en Irak n'ont rien changé sur ce point. L'époque des consultations réciproques obligatoires au sein de l'Alliance atlantique est révolue, et il est évident a posteriori que l'élargissement de l'OTAN à l'Est a été mené afin de réduire l'influence de ceux qui étaient les alliés des États-Unis depuis l'époque de la confrontation Est-Ouest ${ }^{2}$.

C'est dans une telle situation que se sont multipliés les appels adressés aux États-Unis pour leur demander de se satisfaire du rôle d'hégémon 
bienveillant qui aurait été le leur jusqu'alors et de ne pas ambitionner celui de force impériale. Pour donner de l'importance à de tels avertissements, on a rappelé les risques incontrôlables que couraient les empires, le danger que représentait une expansion excessive, et qu'aucun des empires du passé n'avait pu éviter le déclin. "Tandis que par le passé, le pouvoir des États-Unis a été hégémonique et que, de ce fait, on l'a en règle générale accepté, voire considéré comme légitime à l'étranger, il se fonde aujourd'hui sur la puissance des armes » : tel est l'avis de Michael Mann, un Britannique qui enseigne aux États-Unis. « Cela mine le caractère hégémonique et la prétention à être un "empire bienveillant"’. » Celui qui tenterait d'échanger la position hégémonique contre une position impériale courrait non seulement le risque d'échouer dans cette tentative, mais également celui de perdre sa position hégémonique. Les notions de position hégémonique et d'empire ont été opposées l'une à l'autre en de multiples variations, pratiquement toujours assorties de la recommandation selon laquelle il vaudrait mieux rester hégémon que de viser la domination impériale.

D'un seul coup, ce débat, initié au sujet des intérêts et des intentions des États-Unis dans la région du Golfe, a été mené avec une pléthore d'arguments et de comparaisons tirés de l'histoire dans le but de rendre familiers et clairs les aspects nouveaux et agaçants de la politique des États-Unis et des constellations politiques mondiales. L'histoire de l'Empire romain est devenue le repoussoir sur l'arrière-fond duquel ont été jugés tant les possibilités s'offrant à la politique américaine que les risques qu'elle courait ; la structure de l'Empire britannique a été le modèle à l'aune duquel on a mesuré les défis impériaux et les capacités nécessaires aux États-Unis pour les relever; enfin, on a brandi la chute de l'Union soviétique, survenue une bonne décennie auparavant, pour illustrer les dangers liés à l'expansion excessive d'un empire, dangers dont les ÉtatsUnis étaient menacés s'ils continuaient sur la voie qu'ils avaient

« De tous les empires de l'histoire, le plus complet le plus achevé est l'Empire romain. II a duré des siècles, et surtout il a, pendant de longues périodes, imposé la Pax romana à l'intérieur d'immenses frontières admirablement défendues. "Immensa pacis romanae majestas". La seule comparaison qui venait à l'esprit concernait l'Empire soviétique, inattaquable, où régnait une "paix soviétique". Or tout paraît indiquer qu'il s'effondre. » Jean-Baptiste Duroselle, Tout Empire périra. Théorie des relations internationales, Paris, Armand Colin, 1992 [1981], p. 295-296. 
« Encore une fois, il ne s'agit en rien de se mettre à chanter les louanges de la tradition yankee ; il s'agit de reconnaître une forme différente, qui, de fait, coïncide [avec le nouveau globalisme]. Nous l'appelons impériale, et si nous la rattachons à l'empire, c'est en grande partie parce qu'elle correspond en plusieurs points à la Rome antique. Bien sûr, si les pères fondateurs des États-Unis s'intéressaient beaucoup à Rome, c'était justement dans cette perspective. Dans une certaine mesure, la notion romaine de souveraineté incluait de la même manière les populations extérieures, les identités hybrides à l'intérieur de Rome, les frontières ouvertes en expansion, ce genre de choses. Les pères fondateurs adoraient dire que les États-Unis devaient être une nouvelle Rome et une nouvelle Jérusalem. Ils avaient certainement tout cela en tête. En tout cas, il ressort de tout ça que l'histoire des États-Unis, et en particulier l'histoire constitutionnelle des États-Unis nous a paru un excellent moyen pour comprendre ce qui se passe aujourd'hui. »

M. Hardt, « Empire », art. cit.

empruntée $e^{4}$. Mais on a recouru à ces remontrances et autres comparaisons historiques plutôt par association que de façon systématique pour étayer pratiquement sans exception des positions adoptées largement en amont. Elles ont davantage servi à illustrer des argumentations en s'appuyant sur l'histoire qu'à vérifier, en s'appuyant de façon fructueuse sur l'expérience, ce qu'il nous est possible d'apprendre à partir de l'histoire de la formation des empires mondiaux du passé. Or la mise en parallèle de l'histoire américaine et de l'histoire romaine s'impose d'autant plus que, depuis leur fondation, les États-Unis se sont réclamés de la République romaine en se plaçant eux-mêmes dans la tradition de celle-ci ${ }^{5}$. Il s'agit dès lors de l'examen critique d'un parallèle qui a occupé depuis toujours une place centrale dans la conscience qu'ont d'elles-mêmes les élites politiques américaines et dans la façon dont elles se perçoivent elles-mêmes. La comparaison avec l'Empire britannique s'impose elle aussi parce que les Américains ont succédé aux Britanniques dans tous les territoires dont ceux-ci se sont retirés après la Deuxième Guerre mondiale et qu'ils ont repris à leur compte la position que ces derniers y occupaient — notamment au Proche Orient, qui a retenu dans le passé récent une grande part de l'attention politique et du potentiel militaire des États-Unis. Enfin, la comparaison avec l'Union soviétique est inévitable, ne serait-ce que parce que les États-Unis et l'Union soviétique se sont disputé pendant quatre bonnes décennies la prépondérance mondiale jusqu'à ce que, épuisés par la course aux armements et par les coûts qu'induisait le maintien de leur empire, les Russes se retirent de la compétition à l'époque de Gorbatchev ${ }^{6}$. 


\section{Empires : la logique de la domination mondiale}

La base de comparaison de ces trois fondations d'empires est néanmoins trop étroite pour mener une analyse fondée des possibilités qui s'offrent à l'empire américain aussi bien que des risques qu'il court. L'empire des tsars russes, l'empire ottoman et l'empire chinois (la puissance impériale dont la longévité a été la plus grande) doivent dans tous les cas être intégrés dans une analyse comparative. Dans le cadre d'une analyse tant de la logique à laquelle obéit l'action des empires que des impératifs auxquels celle-ci est soumise, on ne doit pas non plus négliger la constitution de l'empire mongol au XIII ${ }^{\mathrm{e}}$ siècle. Il s'est rapidement effondré, mais son étendue territoriale en a fait l'un des plus
« On dénombre dans l'histoire plus de soixante méga-empires, dont la grande majorité furent situés dans (ou à proximité de) la région aride transversale à toute l'Afrique et à l'Eurasie, du Sahara à l'ouest au désert de Gobi à l'est. Au titre des exceptions, citons un empire (khmer) en Asie du Sud-est, et le seul empire (inca) des Amériques. L'Europe compterait également trois exceptions : les empires romain et carolingien, ainsi peutêtre que la Lituanie-Pologne, dont la plus grande extension, au XIV siècle, atteignit néanmoins les régions steppiques. Autrement dit, la relation entre la proximité des steppes et l'émergence de méga-empires n'est pas de l'ordre du déterminisme ; cependant, on observe une régularité statistique robuste.

Si la fréquence des méga-empires diminue considérablement à mesure que l'on s'éloigne des steppes afro-eurasiatiques, la raison n'en est pas simplement écologique. D'autres continents sont riches en zones arides, sans que de larges États s'y soient développés, à de rares exceptions près. Le facteur qui paraît le plus important est la proximité de sociétés pastorales.

[...] Afin d'étayer cette vaste généralisation macro-historique, je suggère un modèle du processus social de mise à niveau qui aboutit à l'évolution concomitante des méga-empires agraires et des confédérations impériales nomades, en face à face de part et d'autre de la frontière steppique. Ce modèle d'empires en miroir est fortement influencé par les théories des origines guerrières de l'État. Néanmoins, ce courant important de la théorie anthropologique souffre, à mon sens, de n'avoir pas réussi à intégrer le diagnostic d'Ibn Khaldûn, ni les plus récents développements de la théorie de l'évolution. La formation de l'État suppose force coercition et appât du gain, mais il y a un autre ingrédient crucial : la coopération. »

Peter Turchin, "A theory for formation of large empires », Journal of Global History, 4, 2009, p. 201, 216.

grands de l'histoire universelle : avec une superficie de 25 millions de kilomètres carrés, l'empire mongol n'a été surpassé que par l'Empire britannique qui, à son zénith, couvrait une superficie de 38 millions de kilomètres carrés, distribués sur cinq continents. En s'étendant sur toute l'Eurasie, l'Empire mongol représentait une entité territoriale homogène : 
à l'apogée de sa puissance, il allait de la mer Jaune à l'est jusqu' aux rives de la mer Baltique à l'ouest, et l'occupation mongole n'a épargné que le sous-continent indien, l'Asie du Sud-est (de la Birmanie à l'Indochine) et l'Europe occidentale, centrale et méridionale ${ }^{7}$. En ce qui concerne l'Antiquité, on ne doit pas se contenter de prendre en compte l'Empire romain, mais aussi les empires hellénistiques d'Orient ; au nombre des seaborne empires, il faut, outre les empires britannique et espagnol, s'intéresser à l'empire portugais, le premier empire colonial européen à se constituer et le dernier à disparaitre de la carte politique — il s'agissait assurément depuis la fin du XVIII ${ }^{\mathrm{e}}$ siècle d'un protégé de l'Empire britannique plus que d'une puissance politique autonome ${ }^{8}$.

Cette énumération révèle un problème fondamental dans l'analyse comparative de la logique d'action des empires. Il faut d'abord répondre à la question de ce qu'il faut comprendre sous le terme d'empire - question qu'on pourrait formuler plus précisément encore en affirmant qu'il en va de la différence entre Grand empire et Empire mondial. Il serait certainement plus facile de trouver une réponse si, dans les décennies passées, des études avaient été consacrées aux empires sous l'angle des sciences sociales permettant de définir ce qui fonde la notion d'empire à l'aide de critères sûrs. Ce n'est cependant pas le cas. On note certes la parution d'un nombre considérable de travaux historiques sur quelques empires spécifiques, ainsi que de remarquables études comparatives portant sur l'impérialisme ${ }^{9}$; mais la question de savoir ce qu' est un empire et en quoi il se distingue de l'État territorial tel qu'il s'est formé dans le cadre politique européen n'a été pratiquement pas été traitée. Cela explique que, dans le récent débat au sujet de la politique américaine, la notion d'empire a revêtu une signification plutôt arbitraire se limitant la plupart du temps à dénoncer cette politique. Les sciences politiques n'ont pas défini ses contours à l'aide d'une définition qu'étofferaient des exemples, mais l'ont abandonné aux caprices du journalisme quotidien.

Il n'est pas possible de rattraper d'un seul coup ce qu'un travail de recherche de longue haleine n'a pas fait. Mais tant qu'on ne sait pas de façon claire ce que sont les empires et ce qu'ils ne sont pas, ce qu'il leur revient d'accomplir et en quoi ils se distinguent des autres structures d'organisation du domaine politique, il n'est pas possible, à partir d'une analyse comparative de la fondation des empires, de dégager les éléments à l'aide desquels il serait possible d'analyser le nouvel ordre mondial 
et le rôle qu'y jouent les États-Unis. La logique d'action des empires n'est compréhensible que si l'on a une idée à peu près claire de ce qui caractérise un empire.

\section{Une brève caractérisation des empires}

Pour savoir ce qu'est un empire, il faut d'abord dessiner avec prudence les contours de ce qu'il n'est pas. Premièrement, il faut différencier un empire d'un État, plus précisément de l'État territorial institutionnel, soumis à d'autres impératifs et à d'autres logiques d'action qu'un empire. Cela commence par l'intégration des populations à l'intérieur et va jusqu'à la conception de ce que l'on considère comme frontière. La délimitation des frontières typique des États est claire et nette : elle définit le passage d'un État à un autre. Dans le cas des empires, de telles lignes précises de séparation sont l'exception. Certes, les frontières d'un empire ne se perdent plus aujourd'hui dans le lointain d'un espace où tribus et peuples nomades tantôt suivaient, tantôt défiaient les instructions impériales ; mais même depuis la disparition des espaces dépourvus d'organisation politique dans lesquels les empires classiques ont pu s'étendre, les frontières impériales se distinguent radicalement des frontières étatiques.

Les frontières impériales ne séparent pas des entités politiquement égales, mais représentent plutôt des gradations de pouvoir et d'influence. En outre, et au contraire des frontières étatiques, elles sont semi-perméables. Celui qui veut entrer dans l'espace impérial doit satisfaire à d'autres conditions que celui qui en sort. Cela est à mettre en relation avec l'attractivité économique et culturelle des empires ; plus nombreux sont en général ceux qui veulent y entrer que ceux qui veulent en sortir, ce qui a des conséquences sur la définition des frontières. Les citoyens des États-Unis peuvent voyager et travailler dans le monde entier. Mais ceux qui ne possèdent pas la nationalité américaine ne peuvent pas entrer aux États-Unis comme ils le veulent. C'est en cela que se révèle une différence de statut : les communautés politiques situées aux abords des empires ne jouissent pas de la même dignité que l'empire.

À la semi-perméabilité des frontières impériales correspondent des conditions d'intervention radicalement différentes. Ainsi, depuis la fin du $\mathrm{XIX}^{\mathrm{e}}$ siècle, les États-Unis sont constamment intervenus dans la politique des États d'Amérique centrale et des Caraïbes sans avoir à inclure dans 
leurs calculs le risque que ces États ne leur infligent sur leur territoire des représailles politiques ou économiques — des représailles militaires étant quant à elles totalement exclues. C'est avant tout une telle asymétrie qui différencie les frontières impériales des frontières étatiques. Les empires n'ont pas de voisins qui seraient des semblables, c'est-à-dire qui auraient les mêmes droits, alors que c'est la règle entre les États. Autrement dit : les États existent toujours au pluriel, les empires la plupart du temps au singulier. Qu'il soit réel ou simplement affirmé, ce caractère unique des empires n'est pas sans conséquences sur la façon dont ils procèdent à leur intégration intérieure. Du fait de la concurrence des autres États, les États intègrent leurs populations de façon égale - en leur accordant des droits égaux, qu'elles habitent au cœur de l'État ou dans ses régions frontalières -, ce qui n'est pas le cas des empires. Il y a presque toujours un décalage d'intégration entre le centre et la périphérie, lequel se traduit la plupart du temps par un relâchement des règles de droit et par des possibilités de plus en plus faibles d'être associé aux décisions politiques prises par le centre. Dans le cas des États-Unis, cela se vérifie dans tous ceux des territoires qui, bien que soumis à leur influence, n'ont pas eu la possibilité d'y être intégrés en qualité d'État fédéré. Les exemples ne manquent pas dans les Caraïbes.

Les frontières impériales peuvent être une alternative à celles des États. Les frontières qui, en Europe, séparaient les empires coloniaux européens les uns des autres étaient des frontières étatiques, tandis qu'en Afrique et en Asie les frontières qui les séparaient de leurs voisins (la plupart du temps, il s'agissait de vagues fédérations) étaient des frontières impériales. Ces deux types de frontières différaient fortement les uns des autres et révélaient ce qu'il y avait de l'autre côté : un empire ou un État. Mais les frontières impériales peuvent aussi se superposer aux frontières étatiques et, ce faisant, les consolider. La frontière étatique qui séparait autrefois la République fédérale d'Allemagne de la RDA était également la frontière occidentale de l'Empire soviétique : c'est cette concordance qui a conféré à cette frontière le caractère particulier lui permettant d'entrer dans l'Histoire. Depuis que, dans son ensemble, la surface habitable de la Terre est organisée politiquement en États, il n'y a plus entre les deux types de frontières qu'une relation de complémentarité n'offrant aucune alternative : les structures impériales se superposent à l'ordre des États, mais ne s'y substituent plus. Identifier les empires n'en est que plus 
difficile. Quand on pense le statut d'empire comme simple alternative au statut d'État, on en arrive à la conclusion qu' aujourd'hui, il n'y a plus d'empires. En revanche, quand on part du principe que les structures impériales se superposent aux États, il devient possible de dégager des ensembles d'influence et de pouvoir qui ne sont pas identiques à l'ordre des États. La situation-limite propre aux empires a pour conséquence que l'on identifie les structures impériales plutôt dans le domaine informel. Les frontières des États font souvent concorder les frontières politiques et économiques, linguistiques et culturelles, ce qui leur confère leur force, mais les rend dans le même temps rigides et incapables de s'adapter. En revanche, les frontières impériales peuvent être caractérisées comme un enchevêtrement dans lequel les frontières économiques et politiques sont distinctes, les différences culturelles sont graduées et les différences linguistiques absolument dépourvues de signification, ce qui prive les frontières des empires de leur caractère formel et augmente leur capacité à s'adapter.

« Certains empires des temps modernes étaient à l'évidence liés au plan généalogique, ou bien se trouvaient au croisement de plusieurs aires culturelles distinctes : ainsi les Ottomans, les Safavides ou encore les Moghols, qui semblent même avoir formé une sphère unique de circulation pour les élites, si l'on considère les calligraphes, les mystiques soufis, les militaires ou les poètes ».

Sanjay Subrahmanyam, « Par-delà l'incommensurabilité : pour une histoire connectée des empires aux temps modernes », Revue d'histoire moderne et contemporaine 5/2007 (n54-4bis), p. 36.

Par ailleurs, on doit deuxièmement dessiner les contours de l'empire au regard des structures hégémoniques de domination — à quoi il faut ajouter le flou caractérisant la distinction entre domination hégémonique et domination impériale. Il est néanmoins pertinent de différencier les deux. L'hégémonie est la prédominance exercée à l'intérieur d'un groupe d'acteurs politiques ayant formellement les mêmes droits, tandis que le statut d'empire abolit cette égalité, au mieux formelle, et réduit ceux qu'elle a soumis à un statut d'États clients ou de satellites. De façon plus ou moins visible, ils se retrouvent dépendants à l'égard d'un centre.

Dans les décennies passées, on s'est servi de l'opposition entre empire et hégémonie pour décrire la position de l'Union soviétique dans le pacte de Varsovie et celle des États-Unis dans l'OTAN. L'Union soviétique était entourée d'États satellites dont les mouvements étaient contrôlés par le centre ${ }^{10}$, tandis que l'OTAN était considérée comme un système 
d'alliés ayant par principe les mêmes droits et à l'intérieur duquel une place prépondérante revenait aux États-Unis en leur qualité d'allié de loin le plus important et le plus puissant — ne serait-ce que parce que c'était celui qui, par principe, pourvoyait le poste de commandant en chef des troupes, tandis qu'il revenait aux autres États de pourvoir celui de secrétaire général. Cette opposition entre Pacte de Varsovie et OTAN permet de voir que dans la confrontation Est-Ouest, la différenciation entre hégémonie et empire revêtait une charge idéologique.

La transformation de la confédération maritime de Délos en thalassocratie athénienne est un exemple qui, du fait de l'éloignement dans le temps, permet d'illustrer sans la moindre arrière-pensée politique la différence entre hégémonie et empire. Dans la confédération maritime originelle, dirigée contre la domination perse sur la côte occidentale de l'Asie mineure et dans la mer Égée, tous les partenaires avaient les mêmes droits. Certes, dès le début, leurs contributions respectives n'étaient pas identiques : alors que certains se contentaient de verser de l'argent et que d'autres fournissaient quelques navires, l'essentiel du contingent de la flotte de guerre provenait toujours d'Athènes ${ }^{11}$.

L'inégalité effective des contributions et des capacités n'est pas restée sans conséquences sur la constitution interne de la confédération qui, d'une hegemonía, s'est transformée en arché : la prépondérance s'est muée en domination ${ }^{12}$. Athènes pourvoyait le poste de commandant des forces armées et celui de trésorier de l'alliance, déterminait le niveau des contributions, dominait les tribunaux de commerce et a réussi à imposer ses poids et mesures à toute la confédération. En outre, en entretenant des garnisons dans les villes de ses alliés, elle est parvenue à s'ingérer dans leurs affaires intérieures. Enfin, elle a déplacé de Délos à Athènes le trésor de la confédération et n'a plus fait prêter le serment sur " Athènes et ses alliés », mais sur « le peuple athénien »; elle a attribué à l'assemblée populaire athénienne la décision de déclarer la guerre et de signer la paix qui était une prérogative de l'assemblée de la confédération. Lorsque les Corinthiens ont voulu entraîner la ligue du Péloponnèse à déclarer la guerre à Athènes, ils ont affirmé que l'hégémon était devenu un despote ${ }^{13}$.

Il est tentant de décrire le repositionnement des États-Unis à l' " Ouest » avec la transformation de la confédération maritime de Délos en thalassocratie athénienne en arrière-fond. Du point de vue de l'étendue spatiale et de la durée, celle-ci n'a certes pas été un véritable empire, mais on peut 
y observer en miniature de nombreux éléments typiques de la politique impériale - et plus particulièrement parce que ce développement a été décrit par Thucydide d'une façon qui a fait école. C'est pourquoi nous parlerons toujours par la suite de la domination maritime athénienne, même si on ne peut la ranger sous la notion d'empire que de façon fragmentaire.

Enfin, et troisièmement, il faut dessiner les contours de l'empire pour le différencier de ce que, depuis le XIX ${ }^{\mathrm{e}}$ siècle, on appelle l'impérialisme. La différence entre les théories de l'empire et de l'impérialisme permet tout d'abord de se dégager de la perspective normative de pratiquement toutes les théories de l'impérialisme et de jeter un regard analytique et descriptif sur les impératifs d'action qui sont ceux des empires. De plus, la notion d'impérialisme et les théories qui l'accompagnent conçoivent fondamentalement la formation d'empires comme un processus se déroulant du centre vers la périphérie, ce qui suppose une direction de développement à sens unique faisant obstacle à l'observation des empires réels.

Dans le terme «impérialisme », on trouve la volonté d'établir un empire ; il importe peu que celle-ci soit nourrie par des motifs politiques ou économiques - même si ce n'est pas la seule, c'est la raison déterminante pour la formation d'un empire. À l'opposé, on trouve le mot d'esprit de l'historien britannique John Robert Seeley qui, en 1883, a déclaré que l'Empire britannique s'était développé « in a fit of absence of mind $»$ : dans un moment d'absence ${ }^{14}$. Cette formulation, par son unilatéralisme stratégique (Seeley voulait appeler à mener une politique consciemment impérialiste parce qu'il craignait que l'Empire britannique ne fasse les frais de l'expansion des grandes puissances naissantes qu'étaient les États-Unis et la Russie), met précisément en évidence combien les théories de l'impérialisme exagèrent la volonté consciemment délibérée des acteurs impliqués d'une manière ou d'une autre dans l'histoire de la constitution des empires. En général, aucune stratégie d'envergure n'a jamais présidé à la constitution d'un empire. La plupart des empires doivent leur existence à un mélange de hasards et de décisions individuelles prises souvent par des gens qui souvent n'avaient politiquement aucune légitimité à le faire. Vu sous cet angle, presque tous les empires sont apparus « in a fit of absence of mind».

Le regard porté sur le centre, prédominant dans les théories de l'impérialisme, doit être complété par le regard porté sur la périphérie — sur 
la vacance du pouvoir et les dynamiques économiques qu'on y trouve, sur les demandes d'intervention formulées par les vaincus des conflits survenus dans la région, et sur les décisions prises par les responsables locaux. C'est avant tout la fonction initiale de la périphérie dans l'apparition des empires qui s'exprime dans la formule marquante d' " empire sur invitation » récemment utilisée pour décrire l'expansion de la sphère américaine de pouvoir et d'influence ${ }^{15}$. Il y a sans aucun doute une dynamique impériale émanant du centre pour se diriger vers la périphérie et visant à étendre l'espace propre de pouvoir ; à côté de cela, on n'en remarque pas moins la présence d'un courant qui, partant de la périphérie, exerce lui aussi un effet d'entraînement sur l'expansion de l'espace de domination. C'est uniquement au cas par cas que l'on peut déterminer laquelle de ces tendances a été la plus forte. Tandis que les théories de l'impérialisme supposent que la dynamique impulsée par le centre est déterminante ${ }^{16}$, nous partons quant à nous du principe que l'observation précise de la périphérie n'est pas seulement significative au regard des empires du passé, mais aussi pour l'analyse de la politique des États-Unis dans les dernières décennies.

Traduit de l'allemand par Thierry Carpent, avec le concours de Tobias Mörike

\footnotetext{
1. Concernant la période qui a précédé la Troisième Guerre du Golfe, cf. Stefan Aust, Cordt Schnibben (dir.), Irak. Geschichte eines modernen Krieges, Munich, Deutsche Verlags-Anstalt, 2003, et plus particulièrement p. 39 sq. ; Ulrich Tilgner, Der inszenierte Krieg. Täuschung und Wahrheit beim Sturz Saddam Husseins, Berlin, Rowohlt, 2003, p. 17 sq. ; Bernd W. Kubbig (dir.), Brandherd Irak. US Hegemonieanspruch, die UNO und die Rolle Europas, Francfort-sur-le-Main, Campus, 2003, particulièrement p. 9-20 ; Wolfgang Sofksky, Operation Freiheit, Francfort-sur-le-Main, Fischer, 2003, p. 66-74, et Herfried Münkler, Der neue Golfkrieg, Reinbek, Rowohlt, 2003, p. 19-28.

2. Sur l'histoire de l'élargissement de l'OTAN à l'Est et les intentions poursuivies par les parties en présence, cf. Ronald D. Asmus, Opening NATO's Door. How the Alliance remade itself for a new era, New York, Columbia University Press, 2002.

3. Michael Mann, Incoherent Empire, Londres / New York, Verso, 2003, p. 252 ; de même, Ernst-Otto Czempiel, « Pax Americana oder Imperium Americanum ? », Merkur, 57/11 (2003), p. 1003-1014. Le concept remonte à un article de Robert Kagan, «The Benevolent Empire », Foreign Affairs, 76/111 (1998), p. 24-33.

4. Peter Bender, Weltmacht Amerika. Das Neue Rom, Stuttgart, Dt. Taschenbuch-Verlag, 2003, dresse de façon conséquente un parallèle entre les États-Unis et l'Empire romain ; la désignation de « nouvelle Rome» (New Rome) est appliquée aux États-Unis depuis le milieu du XIX ${ }^{\mathrm{e}}$ siècle : cf. Heinz Gollwitzer,
} 


\section{Empires : la logique de la domination mondiale}

Geschichte des weltpolitischen Denkens, Göttingen, Vandenhoek \& Ruprecht, 1972, p. 489 sqq. Une comparaison sous-jacente avec l'efficacité des formes britanniques de domination impériale parcourt l'argumentation de M. Mann, op. cit. Emmanuel Todd, Après l'empire. Essai sur la décomposition du système américain, Paris, Gallimard, 2002, présente la chute de l'Union soviétique comme destin prospectif des États-Unis ; on trouve une série de réflexions équilibrées dans une comparaison des ÉtatsUnis avec les Empires romain et britannique chez Niall Ferguson, Colossus. The Price of America's Empire, New York, Penguin, 2004, p. 19 sqq., p. 34, p. 44 sqq.

5. Cf. Gordon S. Wood, The Creation of the American Republic. 1776-1787, Chapel Hill, University of North Carolina Press, 1969, particulièrement p. 48 sqq. ; Carl J. Richard, The Founders and the Classics. Greece, Rome and the American Enlightenment, Cambridge / Londres, Harvard University Press, 1994. Dès le début, la fière prétention à avoir repris et continué la tradition de la Rome républicaine s'accompagne du regard critique porté sur le passage de la république à l'empire. L'hypothèse, reprise de l'historiographie romaine, qu'un tel changement serait dû à la décadence des mœurs est reportée sur l'Empire britannique : c'est dans cette mesure que l'indépendance des États-Unis, acquise à l'issue de durs combats contre la Grande-Bretagne, a toujours été considérée comme ayant permis de sauver la république face au danger que représentait le passage à l'empire, cf. Bernard Baylin, The Ideological Origins of the American Revolution, Cambridge / Londres, Harvard University Press, 1967, p. 31 sqq. et aussi G. S. Wood, op. cit., p. 35 sq.

6. À ce sujet, et de façon détaillée, cf. Vjačeslav I. Dašičev, Moskaus Griff nach der Weltherrschaft. Die bitteren Früchte hegemonialer Politik, Hambourg, Mittler, 2002, p. 41 sqq. et p. 511 sqq.

7. Pour comparer les Empires mongol et britannique, cf. Hansgerd Göckenjan, « Weltherrschaft oder Desintegration? Krise und Zerfall des Mongolischen Großreichs », dans Richard Lorenz (dir.), Das Verdämmern der Macht Vom Untergang Großer Reiche, Francfort-sur-le-Main, Fischer-Taschenbuch-Verl., 2000 ; au sujet de l'expansion de l'Empire mongol, cf. Michael Weiers, « Geschichte der Mongolen », dans Arne Eggebrecht (dir.), Die Mongolen und ihr Weltreich, Mayence, P. von Zabern, 1989, p. 45.

8. Stefan Breuer, Imperien der Alten Welt, Stuttgart, W. Kohlhammer, 1987, p. 122-158, illustre de façon claire la signification de l'Empire achéménide et de ses successeurs hellénistiques en leur qualité d'empire intermédiaire reliant l'espace méditerranéen et l'Asie ; David B. Abernethy, Dynamics of Global Dominance. European Overseas Empires. 1415-1980, Londres / New Haven, Yale University Press, 2000, p. 45 sqq. et Wolfgang Reinhard, Kleine Geschichte des Kolonialismus, Stuttgart, A. Kröner, 1996, p. 25 sqq. traitent de façon détaillée le rôle initial du Portugal dans la formation des empires coloniaux européens.

9. Voir à ce sujet la vue d'ensemble qu'en donne Wolfgang J. Mommsen, Imperialismustheorien. Ein Überblick über die neueren Imperialismustheorien, Göttingen, Vandenhoeck \& Ruprecht, 1977.

10. Que je sache, la comparaison du pouvoir impérial avec le soleil et ses satellites ne relève assurément pas du statut impérial militaire, mais économique. C'est ainsi qu'au début du XIX ${ }^{\mathrm{e}}$ siècle, le banquier Nathan Rothschild a déclaré devant la Chambre des Communes que « Londres était la capitale mondiale de la finance et que même les grandes affaires commerciales devaient plus ou moins nécessairement être conclues sous l'influence de ce qui était le centre du système financier autour duquel se mouvaient des États moins fortunés tout comme le font les petits astres autour du soleil, et duquel ils devaient s'estimer heureux de pouvoir tirer leur éclat et leur subsistance. » Cité par H. Gollwitzer, op. cit., vol. 1, p. 505 sqq.

11. Cf. Wolfgang Schuller, Die Herrschaft der Athener im Ersten Attischen Seebund, Berlin, Walter de Gruyter, 1974, p. 54 sqq.

12. Heinrich Triepel, Die Hegemonie. Ein Buch von führenden Staaten, Stuttgart, W. Kohlhammer, 1938, a qualifié ce phénomène d' « hégémonie absorptive ».

13. Cf. S. Breuer, op. cit., p. 140-147 ; pour un point de vue détaillé, voir Karl-Wilhelm Welwei, Das klassische Athen. Demokratie und Machtpolitik im 5. und 4. Jahrhundert, Darmstadt, Primus, 1999, p. 77-139; au sujet de la transformation de l'hegemonía en arché, H. Triepel, op. cit., p. 343 sqq. ; au sujet de la transformation effective de la confédération, ibid., p. 377 ; W. Schuller, op.cit., p. 153-165, offre une présentation détaillée de la domination athénienne. 


\section{Labyrinthe, $n^{\circ} 35$}

14. Cité d'après Niall Ferguson, Empire : How Britain Made the Modern World, Londres, Allen Lane, 2003, p. 246.

15. Cf. Charles S. Maier, «Die Grenzen des Empire », dans Ulrich Speck, Nathan Sznaider (dir.), Empire Amerika. Perspektiven einer neuen Weltordnung, Munich, Deutsche Verlags-Anstalt, 2003, p. 128.

16. Pour être exhaustif, il convient de dire que les «théories de l'impérialisme s'orientant selon les périphéries », une sous-catégorie des théories de l'impérialisme, ont très bien pris en compte le rôle de la périphérie dans la constitution des empires. Elles partent du principe que « les actions impérialistes des grandes puissances sont en règle générale déclenchées par des situations de crise dans les pays du Tiers monde » : W. J. Mommsen, op. cit., p. 80-90, ici p. 81. 